\title{
Globalización y desarrollo en la región de Olocuilta y San Luis Talpa
}

PATRICIA Fuentes:

\section{Introducción}

El Salvador es el país más pequeño de Centroamérica con una superficie de $20,877 \mathrm{~km}^{2}$ y una población estimada de 6,3 millones de habitantes a comienzos del 2001 (CEPAL, 2000), alcanzando una densidad superior a los 300 habitantes por $\mathrm{km}^{2}$, que lo convierte en el país más densamente poblado de América Latina. Alrededor del $60 \%$ de la población vive en zonas rurales y más del $80 \%$ de los productores agrícolas trabajan en explotaciones de superficie inferior a 3 ha. Según datos tomados del Informe sobre Desarrollo Humano 2001, "entre 1975 y 1980, el índice de desarrollo humano (IDH) se mantuvo inalterado, pese a que el PIB per cápita estuvo creciendo a tasas relativamente altas" (PNUD, 2001:92). Esto contribuyó muy poco a disminuir los niveles de pobreza y al acceso de la población a los servicios básicos. Entre 1980 y 1990, el PIB per cápita decreció, sin embargo el IDH aumentó, por la cobertura proporcionada a los servicios de salud. El retroceso del PIB per cápita en los años ochenta se debió,

- Trabajo elaborado para el Diplomado en Gestión Económica Local. FLACSO, Costa Rica/Instituto de Estudios Sociales de la Haya, Holanda. Patricia Fuentes es profesora del Departamento de Organización del Espacio de la UCA.

Globalización y desarrollo en la región de Olocuilta y San Luis Talpo 
fundamentalmente, al conflicto armado, pero su bajo crecimiento en los períodos de relativa normalidad es producto de las debilidades de los diferentes modelos de desarrollo practicados. En la década de los noventa, el informe indica que, tanto el PIB como el IDH aumentaron, logrando disminuir la pobreza y mejorar el acceso a los servicios sociales básicos. Sin embargo, aún no se logra avanzar en el crecimiento económico sostenido del país.

Después de los dos terremotos de enero y febrero de 2001, el nivel de pobreza en el país pasó del $47.3 \%$ al $49.7 \%$. Según estimaciones de la Fundación Salvadoreña para el Desarrollo Económico Social (FUSADES), este incremento de 2.4 puntos porcentuales ocurrió principalmente en el nivel de pobreza extrema. Como pobreza extrema se entiende a aquellas personas cuyos ingresos no alcanzan a cubrir la canasta básica. FUSADES realizó estas estimaciones con base en la pérdida por Departamento, tanto de los activos destruidos como por los ingresos que se han dejado de percibir, principalmente por el desempleo originado. Los Departamentos en los que más activos se destruyeron fueron San Vicente, La Paz, Cuscatlán y Usulután.

El propósito de este trabajo es identificar cuales han sido los impactos de la globalización en el desarrollo de dos municipios de la región costera central de El Salvador en los años recientes, Olocuilta y San Luis Talpa, ubicados en el departamento de la Paz, uno de los mas afectados por los terremotos del 2001. Se procura identificar en qué forma la región ha sido impactada por este proceso, evaluando al mismo tiempo desde una óptica realista las posibilidades y riesgos que plantea el proceso de globalización.

La primera parte se dedica al análisis de la dinámica socioeconómica del país, las políticas de ajuste económico y la reforma del Estado impulsadas en los años 90 , y su relación con el proceso de globalización, lo que permitirá contextualizar las posibilidades de desarrollo de los municipios de Olocuilta y San Luis Talpa.

La segunda parte se dedica al análisis de la dinámica socio-económica de la región, de la propuesta de la Comisión Nacional de Desarrollo (CND) y las inversiones recientes vinculadas al proceso de globalización efectuadas en la misma.

La tesis central de este trabajo es que las economías comunitarias que no estén incluidas en las actuales políticas públicas del país y que 
no cuenten con una importante inversión privada, ambas en estrecha combinación, serán incapaces de integrarse y aprovechar los efectos positivos de la globalización e incidir en los negativos, y por lo tanto difícilmente lograrán un desarrollo sostenido.

\section{Marco teórico. La globalización y el contexto nacional}

Para elaborar el marco teórico se han utilizado algunos conceptos que se derivan de las consideraciones de algunos teóricos e investigadores, con relación a temas como: globalización, desarrollo económico local y descentralización.

El término globalización se refiere a un fenómeno que ha adquirido diversas definiciones y puntos de vista antogónicos. Algunos teóricos consideran que la globalización es: “...un fenómeno multidimensional aplicable a variedad de formas de acción social -económica, política, legal, cultural, militar y tecnológica- y sitios de acción social, como del medioambiente"(Perraton, 1997: 258). En sus palabras este sostiene que la globalización es un proceso nuevo y que no sabemos que va a pasar. Otros opinan que: “...la globalización no sólo homogeniza y nos vuelve más próximos, sino que multiplica las diferencias y engendra nuevas desigualdades..." (García, 1999: 50), debido a que consideran que suscita una mayor desigualdad dentro de cada país y entre los distintos países, amenaza el empleo y las condiciones de vida y obstaculiza el progreso social. Un tercer enfoque enfatiza el razonamiento de la exclusión y diferenciación: “...el peligro más claro para la viabilidad de las comunidades no es la globalización, sino el aislamiento y el proteccionismo. En la economía global, aquellas instituciones que están fragmentadas y aquellos pueblos u organizaciones que no estén conectados y estén aislados se encuentran es desventaja. (Kanter, en De Campos G., 2001:3) y un último enfoque sostiene que la "Globalización es un espacio de poder y de toma decisiones" (Sojo, 2001) el cual induce a cambios económicos; genera o reposiciona subjetividades (aparecimiento y desaparecimiento de actores locales); y modifica el locus de la vida social.

Estos conceptos son importantes, porque nos darán una visión más amplia del impacto de este fenómeno en las economías nacionales y locales de El Salvador. Así como cuales son las consecuencias de la internacionalización a nivel regional y local. 
Se entiende el desarrollo económico local "como un proceso de crecimiento y cambio estructural que mediante la utilización del potencial de desarrollo existente en el territorio conduce a la mejora del bienestar de la población de una localidad o una región. Cuando la comunidad local es capaz de liderar el proceso de cambio estructural, la forma de desarrollo se puede convenir en denominarla desarrollo local endógeno. (Vásques, en CEPAL/GTZ, 2000:5).

Lograr el desarrollo económico local puede implicar un cambio fundamental en los actores y en las actividades relacionadas con el desarrollo, el cual trata de asegurar un nivel de empleo adecuado y para ello es necesario captar inversiones (nacionales e internacionales) que garanticen la equidad y el crecimiento económico sostenible y sustentable. Esto conlleva a la creación de nuevas instituciones, capacitación del recurso humano y principalmente mejorar la calidad de vida de la población. En este sentido se pretende hacer una reflexión sobre el desarrollo local y sobre los efectos de la globalización en la región en estudio.

La descentralización es definida (Carrera, 2001:2) como "la transferencia de responsabilidades para llevar a cabo la planificación, generación, distribución y administración de recursos, por parte del gobierno nacional hacia: i) oficinas desconcentradas del gobierno nacional; ii) diferentes niveles de gobierno; iii) corporaciones o autoridades públicas semiautónomas; iv) autoridades regionales o funcionales y v) organizaciones no gubernamentales privadas o voluntarias". También la define como "la transferencia de poder y responsabilidades hacia gobiernos locales electos los cuales los ejercen con un grado considerable de autonomía".

Estos conceptos serán básicos en este trabajo para comprender la implicación que ha tenido la distribución del poder de decisión sobre los municipios en estudio y verificar si estos procesos han sido implementados o todavía se mantienen en el discurso político.

Se puede afirmar que en El Salvador se han experimentado fuertes cambios debido al proceso de globalización desde hace mas de una década, provocados por la reorientación de las inversiones del sector privado nacional y extranjero, e impulsados por la creación de políticas de estabilización y ajuste estructural de la economía y la reforma del Estado. La globalización, ha permitido que el país se beneficie al 
tener mayor acceso a los flujos de capital y a la tecnología y mercados de exportación más amplios.

Otro factor importante ha sido la dotación de infraestructura por parte del estado, con el propósito de estimular la inversión en localidades con altos índices de pobreza. El desafío es buscar y conciliar nuevas formas de relación entre los niveles internacional y nacional que permitan que los efectos positivos lleguen al nivel del desarrollo económico local.

\section{Contexto general. Evolución de la economía salvadoreña}

Entre los años cincuenta y sesenta, la economía salvadoreña experimentó importantes transformaciones en su estructura productiva. Las políticas económicas buscaban favorecer el crecimiento de actividades industriales que permitieran la sustitución de importaciones y que, al mismo tiempo, diversificaran la oferta productiva. De esta forma, la economía del país experimentó una modificación que incrementó la participación del producto industrial en el total nacional. Este cambio estuvo favorecido por el crecimiento de la economía mundial de posguerra y por el desarrollo del Mercado Común Centroamericano (MCCA), utilizando elevados niveles de protección contra la producción proveniente fuera de la región. A estas medidas comerciales se agregaron un conjunto de estímulos de carácter fiscal y crediticio, que permitieron un ambiente favorable para la inversión externa en actividades industriales. Los efectos de esta estrategia en el país, fueron un fuerte estado nacional y un alto grado de centralismo, con aumento de los niveles de pobreza.

Las actividades industriales se orientaron hacia el mercado interno y centroamericano, estas empresas industriales permitieron generar numerosas fuentes de empleo en el Área Metropolitana de San Salvador, la cual presentaba un creciente dinamismo. Sin embargo, estas no se preocuparon por expandir y mejorar la calidad de sus productos a mercados fuera de la región centroamericana, siendo altamente dependientes de insumos intermedios y materias primas importadas.

Este fue uno de los principales problemas estructurales que agudizaron la situación de crisis de principios de los ochenta en El Salvador, que se vio aumentada a raíz del conflicto bélico. Otro de los factores que contribuyeron a la crisis fue "un aumento en las tasas de

Globalización y desarrollo en la región de Olocuilto y San Luis Talpa 
interés a nivel mundial, una caída de productos comerciales (petróleo), aumento de los déficits presupuestales y una incapacidad para cumplir con el servicio de la deuda externa" (Carrera, 2001:2,3). Tal como menciona Carrera, en los noventa se hizo evidente el fracaso de los gobiernos nacionales en lograr el desarrollo económico, bienestar social, equidad regional, autonomía local y estabilidad macroeconómica, en este contexto El Salvador no fue la excepción.

Posteriormente en los años noventa, surgen en El Salvador cambios en las políticas de crecimiento económico, como la reforma del Estado la cual intenta fomentar el crecimiento económico, siendo su rasgo distintivo la privatización y el impulso a una economía abierta, caracterizada por la apertura al comercio internacional y a las inversiones. De este esquema se pasa a un modelo de crecimiento abierto e internacionalizado y descentralizado. (La Reforma del Estado: Reto de la Democracia: www.clad.org.ve/0029803.html). El actual gobierno no ha dado marcha atrás a este proceso, sino todo lo contrario, se han elaborado reformas en sus políticas para fortalecer las economías y el sistema financiero, con el propósito de lograr un crecimiento a mediano y corto plazo.

\section{Reestructuración económica y reforma del Estado en la década de los noventa}

"Durante los noventa, una vez que se reconoció lo negativo de los resultados económicos y políticos derivados del modelo de Estado neoliberal; la concepción de la reforma administrativa se amplió, trasformándose en parte sustancial de la reforma del Estado. Esta última se ha transformado en un amplio proceso de reflexión de la relación Estado y sociedad, que además, de contemplar la transformación de la administración pública y la burocracia, también incorporará un replanteamiento del papel de la sociedad civil y de su participación.

La reforma administrativa se considera ahora como la transición de un modelo administrativo burocrático hacia uno denominado de gestión pública. Esta última, tiene como reto no sólo responder a la crisis de los ochenta, sino también adaptarse al proceso de globalización". (Carrera, 2001:2,3)

A continuación se mencionan algunos programas de reestructuración de la economía y de reforma del Estado que buscan una mejor inserción de la economía salvadoreña en el mercado mundial. 
Programa de reestructuración de la economía

Este se basa en la liberalización de precios, tarifas y tasas de interés; estabilización macroeconómica (control de la inflación y estabilidad del tipo de cambio); modernización tributaria; apertura comercial; cambios en la estructura de las exportaciones; modernización tecnológica; promoción de inversiones extranjeras y cambios en la relación capital/trabajo.

\section{Programa de reforma del Estado}

El programa de reforma del Estado se basa principalmente en la reducción y modernización del aparato estatal, en la privatización de los activos estatales, la descentralización y modificación en las regulaciones.

\section{Programa de mercados financieros y de capitales}

Este se basa en favorecer la productividad. El asesoramiento y la asistencia técnica al sector financiero, que es uno de los principales elementos de apoyo.

El Banco Mundial está apoyando a El Salvador en el proceso de reforma. Primero, a través del Programa de Competitividad, el cual tiene por objetivo: (i) mejorar las condiciones para la actividad económica adoptando una nueva ley de inversiones; medidas legislativas para la protección del consumidor; prestación de servicios de infraestructura por el sector privado; profundización de los mercados financieros y de capitales; eliminación de las barreras al comercio que aún persisten; adopción de un criterio de agrupación de sectores clave; y reforma de los registros jurídicos; (ii) mejora de la absorción de tecnología mediante el aumento de la calidad y el fortalecimiento de las políticas de capacitación técnica; y (iii) logro de un consenso nacional sobre los planes de competitividad por conducto de un programa de información pública y viajes de estudios para evaluar la experiencia internacional.

El informe sobre Desarrollo Humano presentado por el PNUD en el 2001, afirma que a pesar de la opinión de Heritage Foundation de que "la agresividad del proceso salvadoreño de reformas económicas ha sido tal que en su último informe aparece clasificado en el primer 
lugar de América Latina y en una de las mejores posiciones mundiales, a partir del índice de libertades económicas (ILE). ${ }^{\prime}$..., el hecho de que El Salvador haya avanzado en materia de libertades económicas no es condición suficiente para que, como consecuencia del mayor espacio al libre mercado, también fuera líder en competitividad." (PNUD, 2001:95). En el cuadro siguiente se muestra cuales son las áreas de debilidad en términos de competitividad en el país.

\section{Cuadro 1}

Areas débiles en términos de competitividad internacional en El Salvador

\begin{tabular}{|c|c|}
\hline Sector & Área de debilidad \\
\hline $\begin{array}{l}\text { Operaciones y estrategias } \\
\text { de las empresas }\end{array}$ & $\begin{array}{l}\text { o Importancia del entrenamiento } \\
\text { a ejecutivos } \\
\text { o Capacidad de innovación } \\
\text { o Control de la distribución } \\
\text { internacional }\end{array}$ \\
\hline Estado de la infraestructura física & $\begin{array}{l}\text { o Estado de las carreteras } \\
\text { o Capacidad de generación de } \\
\text { energía eléctrica } \\
\text { o Infraestructura Ferroviaria } \\
\text { o Desarrollo red logística interna }\end{array}$ \\
\hline $\begin{array}{l}\text { Infraestructura administrativa } \\
\text { y de información }\end{array}$ & $\begin{array}{l}\text { o Imparcialidad en reclamos } \\
\text { frente al Estado } \\
\text { o Disponibilidad de información } \\
\text { sobre negocios }\end{array}$ \\
\hline $\begin{array}{l}\text { Disponibilidad de capital } \\
\text { y recurso humano }\end{array}$ & $\begin{array}{l}\text { o Sofisticación del mercado } \\
\text { financiero } \\
\text { o Acceso al mercado de valores } \\
\text { o Disponibilidad de capital de } \\
\text { riesgo } \\
\text { o Calidad de la educación } \\
\text { primaria y secundaria } \\
\text { o Escolaridad de la fuerza de } \\
\text { trabajo }\end{array}$ \\
\hline
\end{tabular}




\begin{tabular}{|c|c|}
\hline Sector & Área de debilidad \\
\hline & $\begin{array}{l}\text { o Calidad y disponibilidad } \\
\text { de científicos e ingenieros } \\
\text { o Calidad escuelas de negocios }\end{array}$ \\
\hline Desarrollo de ciencia y tecnología & $\begin{array}{l}\text { o Gasto en investigación y } \\
\text { desarrollo } \\
\text { o Calidad de las instituciones de } \\
\text { investigación } \\
\text { o Cooperación de empresas y } \\
\text { universidades en investigación }\end{array}$ \\
\hline Condiciones de demanda & $\begin{array}{l}\text { o Exigencia de los compradores } \\
\text { o Desarrollo regulatorio }\end{array}$ \\
\hline $\begin{array}{l}\text { Estrategia, estructura y rivalidad } \\
\text { de las empresas }\end{array}$ & $\begin{array}{l}\text { o Protección a la propiedad } \\
\text { intelectual } \\
\text { o Base de la competencia local }\end{array}$ \\
\hline
\end{tabular}

Fuente: Elaborado con base en "Análisis del Informe Global de Competitividad Microeconómica para Centroamérica" INCAE. 1999.

Segundo, a través del Programa de Mercados financieros y de capitales. Este se basa en favorecer la productividad, asesoramiento y asistencia técnica al sector financiero, siendo uno de los principales elementos de apoyo.

Adicionalmente, el Gobierno ha definido el acceso a la información y a los conocimientos como elemento clave para su estrategia de competitividad y desarrollo social. Las recomendaciones del Banco Mundial son que este programa se acompañe de políticas sociales firmes, para conseguir una integración más plena del programa social y el de competitividad.

Sin embargo, en una entrevista al sr. Arturo Zablah, (ex presidente CEPA) afirmaba que "el país importa mas del doble de lo que esta exportando" y que "se tienen las tarifas de energía eléctrica, agua y combustible más caras de la región centroamericana". Este punto de vista permite evaluar las fortalezas y limitaciones de las políticas implementadas en este período (Canal 12, 2001). 


\section{Dinámica socio-económica de la región e inversiones recientes}

Los municipios de Olocuilta y San Luis Talpa están ubicados en la región paracentral del país, en el corredor que forma la autopista San Salvador-Aeropuerto Internacional El Salvador. Se hará una descripción y breve análisis sobre la dinámica económica producida por los efectos del proceso de globalización en ellos y de la influencia que en esto ha ejercido el cambio en el rol del Estado.

Hasta hace dos décadas ambos municipios se caracterizaban por el predominio de los cultivos agrícolas de subsistencia. Loa caminos de tierra y la carretera principal no reunían las condiciones necesarias de accesibilidad y movilidad, limitando las actividades comerciales y de comunicación.

El municipio de Olocuilta tiene una extensión de $89.68 \mathrm{Km} 2$, la población para 1992 en el área urbana era de 5,004 (31.26\%) y en el área rural de 10,988 (68.71\%). (DIGESTYC,1992).

\section{Cuadro 2}

\section{Característica del territorio y núcleos de población del municipio de Olocuilta}

\begin{tabular}{|c|c|}
\hline \multicolumn{2}{|c|}{ Municipio Olocuilta, categoría ciudad } \\
\hline Características de & $\begin{array}{l}\text { - Existen importantes centros de } \\
\text { comercio de servicios aledaño a la } \\
\text { autopista. } \\
\text { o Existen desarrollos habitacionales de } \\
\text { alta densidad sin los equipamientos } \\
\text { necesarios. } \\
\text { o Se localizan parcelaciones } \\
\text { industriales de gran magnitud. } \\
\text { o Localización de ventas de pupusas } \\
\text { y comida aledañas a la autopista. } \\
\text { o Débil gestión municipal y escasos } \\
\text { recursos económicos. } \\
\text { o Falta de un plan estratégico munici- } \\
\text { pal que involucre a la región. }\end{array}$ \\
\hline Núcleos de población & $\begin{array}{l}\text { o Urbanización Montelimar, Parcela- } \\
\text { ción San Juan de Dios, Parcelación } \\
\text { Mazada y Residencial Edén. }\end{array}$ \\
\hline
\end{tabular}

Fuente: claboración propia. 
El municipio de San Luis Talpa tiene una extensión de $65.96 \mathrm{Km} 2$, la población para 1992 en el área urbana era de 3,258 (21.14\%) y en el área rural de 12,152 (78.86\%). (DIGESTYC,1992).

La tasa de crecimiento anual promedio de este municipio fue de $5.56 \%$ entre 1971 y 1992 , superior a la del AMSS que creció a $3.56 \%$ en el mismo período. Su importancia como centro urbano más cercano al aeropuerto ha crecido y se refleja por el reciente título de ciudad otorgado, según el Decreto 817, de febrero de 2000. (VMVDU, 2000).

Este municipio es el que más refleja los cambios producidos por las políticas e inversiones impulsadas por el gobierno en la zona debido a la construcción del aeropuerto.

\section{Cuadro 3}

Característica del territorio y núcleos de población del municipio de San Luis Talpa

\begin{tabular}{|c|c|}
\hline Municipio San Luis Talpa & \\
\hline Características del territorio & $\begin{array}{l}\text { o Asentamientos territoriales dis- } \\
\text { persos y lotificaciones } \\
\text { habitacionales de alta densidad. } \\
\text { o } \begin{array}{l}\text { Débil gestión municipal y falta } \\
\text { de recursos económicos }\end{array} \\
\text { o Falta de un plan estratégico que } \\
\text { involucre a la Región. } \\
\text { Núcleos de población } \\
\text { o Parcelación Miraflores, Parcela- } \\
\text { ción Santa Cristina y } \\
\text { Lotificación San Luis. }\end{array}$ \\
\hline
\end{tabular}

Fuente: elaboración propia

Ambos municipios están localizados en la zona de influencia a la autopista San Salvador- Aeropuerto Internacional El Salvador, que involucra ocho municipios, de los cuales dos forman parte del AMSS (San Salvador y San Marcos), y dos más (Santo Tomas y Santiago Texacuangos) al AMSS ampliada, pertenecientes todos al Departamento de San Salvador; los otros cuatro municipios (Olocuilta, Cuyultitán, 
San Juan Talpa y San Luis Talpa) forman parte del Departamento de la Paz.

Con la construcción del nuevo aeropuerto en los años 70, localizado en San Luis Talpa se construye una moderna carretera que abre las puertas al surgimiento de nuevas actividades y mercados locales en la región. El aeropuerto ha ejercido desde su construcción un rol regional importante, facilitando el comercio exterior y el turismo, contribuyendo al desarrollo económico de la región.

El Aeropuerto Internacional de El Salvador (AIES), se encuentra localizado en el municipio de San Luis Talpa y entró en operaciones en 1980. Por la misma época se inició la construcción de una nueva carretera para conectar el aeropuerto con la capital del país, lo que abrió las puertas al surgimiento de nuevas actividades y mercados locales en la región. La carretera se proyectó de cuatro carriles con una capacidad para 3,000 vehículos/hora y venía a sustituir la carretera antigua (VMVDU, 2000: 4). Antes de la construcción de la autopista, la región era básicamente de bosques de cafetal en la parte alta y cultivo de algodón en la parte baja.

La autopista se diseño como una vía rápida con accesos controlados y limitados a cinco lugares. Sin embargo, no se previó la demanda que generaría el crecimiento del Área Metropolitana de San Salvador (AMSS), el tráfico que se generaría desde el oriente del país y el tráfico generado por las actividades del Aeropuerto.

$\mathrm{Al}$ inicio de la década de los ochenta, las condiciones de inversión se deterioraron rápidamente. Esto afectó directamente la demanda de pasajeros en el Aeropuerto, al mismo tiempo que la inseguridad afectó la movilidad general. Ambos aspectos conjugados llevaron a que las expectativas en cuanto al volumen de tráfico en la autopista no alcanzaran las proyecciones.

El tráfico internacional aeroportuario, que en 1978 había alcanzado un poco más de 357,000 pasajeros, para 1980 había decrecido en un $26 \%$ a 264,015 pasajeros. El volumen de pasajeros internacionales de entrada, que en 1978 había alcanzado un poco más de 180,000 para 1982 había decrecido en un $36 \%$ a 115,510 pasajeros.(VMVDU, 2000: 4).

Esta región es la mejor conectada del país. La carretera litoral sirve para unir sitios claves, incluyendo la capital, playas, zonas productoras 
y áreas de vivienda. Cuenta, además, con buenas instalaciones eléctricas, telefónicas y la infraestructura adecuada para el funcionamiento de parques industriales. Con la iluminación de la carretera de Comalapa en 1999 y la reconstrucción de la misma a través de la creación del Fondo Vial (FOVIAL) que inició sus actividades en el 2001, se espera una mayor reactivación de las actividades económicas en la región.

Uno de los elementos que ha incidido en el crecimiento industrial y en la ampliación y reactivación de la economía de la región, es la apertura a la economía mundial posibilitada por la construcción del aeropuerto y de la autopista que ha conformado un corredor de desarrollo alrededor del cual se están estableciendo varias zonas francas dedicadas especialmente a albergar empresas maquiladoras de piezas electrónicas para celulares y del sector textil.

Según el análisis de coyuntura económica del último semestre, realizado por la Universidad Centroamericana "José Simeón Cañas" (UCA), la economía salvadoreña al hacer una comparación de las exportaciones de enero a junio de 2000 con las del primer semestre de este año, observa que éstas se han reducido en el 3.1 por ciento, sobre todo en las exportaciones tradicionales como el café, no así las exportaciones no tradicionales como la maquila, que experimenta un crecimiento del 6 por ciento. (ECA, 2001:794)

Otra de las estrategias claves del presente Gobierno consiste en hacer que la base competitiva de El Salvador sea una mano de obra con productividad elevada, es decir, en lograr una ventaja competitiva, más que comparativa. "La competitividad bien entendida en la nueva economía informacional global no pasa fundamentalmente por una reducción de costos sino por un incremento de productividad. Y esa productividad depende, en lo esencial, de tres factores: conectividad, innovación y flexibilidad institucional" (Borja y Castells, 1997:32). Esto constituye la base para comprender que la competencia consiste no solo en reducir costos, sino, en el manejo de los diferentes factores y actores que participan en el proceso.

En el país se registran actualmente once zonas francas, de las cuales dos están localizadas en el municipio de Olocuilta, la zona franca "El Salvador Internacional" y la zona franca "Miramar". En el cuadro se puede apreciar el techo industrial con el que cuenta cada una de ellas: 
Cuadro 4

Zonas Francas

\begin{tabular}{|c|c|c|c|c|}
\hline Nombre & $\begin{array}{l}\text { Área } \\
\text { total }\end{array}$ & $\begin{array}{l}\text { Área } \\
\text { industrial }\end{array}$ & $\begin{array}{l}\text { Área } \\
\text { construida }\end{array}$ & $\begin{array}{l}\text { Fuerza } \\
\text { laboral } \\
\text { potencial }\end{array}$ \\
\hline $\begin{array}{l}\text { El Salvador } \\
\text { internacional }\end{array}$ & $\begin{array}{l}8,345,900 \\
\text { pies cua- } \\
\text { drados }\end{array}$ & $\begin{array}{l}198 \mathrm{mil} \\
\text { metros } \\
\text { cuadrados }\end{array}$ & $\begin{array}{l}42,000 \\
\text { metros } \\
\text { cuadrados }\end{array}$ & $\begin{array}{l}2,474,710 \\
\text { personas } \\
\text { viven en } \\
\text { un radio de } \\
20 \text { millas }\end{array}$ \\
\hline Miramar & 63 acres & $\begin{array}{l}17,5000 \mathrm{mil} \\
\text { metros } \\
\text { cuadrados }\end{array}$ & $\begin{array}{l}9,000 \text { mil } \\
\text { metros } \\
\text { cuadrados }\end{array}$ & $\begin{array}{l}\text { Más de } \\
100 \text { mil } \\
\text { personas }\end{array}$ \\
\hline
\end{tabular}

Fuente: Elaboración propia a partir de PROESA (Agencia de Promoción de Inversioncs)

La meta principal de los empresarios de la región en estudio, es la diversificación de las Zonas Francas, buscando atraer inversionistas para actividades de centros de llamadas y producción de piezas electrónicas. Diversificar los productos que se maquilan dentro de los recintos fiscales, ya que en el país solo la firma "AVX" se encarga de la fabricación de piezas electrónicas para celulares. Tampoco descartan la posibilidad de atraer inversiones para el área de confección y la industria naviera. (PROESA,2001).

Los representantes de las zonas francas aseguran que los empresarios que decidan instalarse en el país no tendrán problemas, ya que se cuenta con suficiente techo industrial en construcción. Según las cifras del Ministerio de Economía, se construyen 70 mil metros cuadrados de techo industrial, y las proyecciones de crecimiento son de 135 mil metros cuadrados, al terminar el año. La entidad espera que se generen 15 mil empleos directos.

La zona franca "El Salvador Internacional" y la zona franca "Miramar", son de las inversiones recientes en el municipio de Olocuilta, lo que está generando y transformando muchas de las actividades locales del municipio y en la región. 
En el cuadro siguiente se observa los porcentajes de PEA para los municipios de Olocuilta y San Luis Talpa.

\section{Cuadro 5}

Distribución porcentual de la Población Económicamente Activa (PEA) y población ocupada de los municipios de Olocuilta y San Luis Talpa, por Área y Sexo, en el año 1992

\begin{tabular}{|c|c|c|c|c|c|c|c|c|c|}
\hline \multirow[t]{3}{*}{ Municipios } & \multirow{2}{*}{\multicolumn{3}{|c|}{ Total }} & \multirow{2}{*}{\multicolumn{3}{|c|}{ Ares }} & & & \\
\hline & & & & & & & \multicolumn{3}{|c|}{ Rural } \\
\hline & Total & Hombres & Mujeres & Total & Hombres & Mujeres & Total & Hombres & Mujeres \\
\hline Olocuilta & $\begin{array}{r}6.6 \\
(6.5)\end{array}$ & $\begin{array}{l}5.0 \\
(4.9)\end{array}$ & $\begin{array}{c}1.6 \\
(1.6)\end{array}$ & $\begin{array}{c}2.2 \\
(2.1)\end{array}$ & $\begin{array}{c}1.5 \\
(1.4)\end{array}$ & $\begin{array}{c}0.7 \\
(0.7)\end{array}$ & $\begin{array}{c}4.4 \\
(4.3)\end{array}$ & $\begin{array}{l}3.5 \\
(3.5)\end{array}$ & $\begin{array}{l}0.9 \\
(0.8)\end{array}$ \\
\hline San Luis Talpa & $\begin{array}{r}5.9 \\
(5.8)\end{array}$ & $\begin{array}{l}4.9 \\
(4.8)\end{array}$ & $\begin{array}{l}1.1 \\
(1.0)\end{array}$ & $\begin{array}{c}1.3 \\
(1.3)\end{array}$ & $\begin{array}{l}1.0 \\
(1.0)\end{array}$ & $\begin{array}{c}0.4 \\
(0.4)\end{array}$ & $\begin{array}{c}4.6 \\
(4.5)\end{array}$ & $\begin{array}{l}3.9 \\
(3.8)\end{array}$ & $\begin{array}{l}0.7 \\
(0.7)\end{array}$ \\
\hline
\end{tabular}

Fuente: Censos Nacionales $\mathrm{V}$ de población y IV de vivienda, DIGESTYC, 1992 (VMVDU, 2000: 22).

Nota: Los datos en paréntesis representan los porcentajes de la población ocupada.

En la distribución porcentual de la población económicamente activa, se puede ver que la participación de los hombres es mucho mayor que la de las mujeres. Es importante señalar que la participación de la PEA del área rural es mayor que la del área urbana.

El $32.81 \%$ del suelo actual del municipio de Olocuilta es de uso habitacional, el $67.75 \%$ es industrial y el $8.43 \%$ es suelo comercial. San Luis Talpa tiene el $36.01 \%$ de uso habitacional y el $17.64 \%$ industrial y el $54.53 \%$ de uso comercial (VMVDU, 2000: 48). El uso de suelo habitacional esta representado principalmente por parcelaciones o lotificaciones de alta densidad.

Actualmente existen proyectos por desarrollarse en ambos municipios que vendrán a cambiar las dinámicas económicas de las localidades y de la región misma (VMVDU, 2000: 56). Los proyectos en mención son para el municipio de Olocuilta los siguientes: (i) introducción de la industria metalmecánica, (ii) desarrollo del parque in- 
dustrial La Esperanza, (iii) construcción de naves industriales Z, (iv) construcción de talleres, (v) construcción de mercado municipal, y (vi) construcción de gasolinera. Para el municipio de San Luis Talpa se proyecta lo siguiente: (i) desarrollo de la parte " $A$ " de las Confecciones Samour, (ii) construcción del Hotel Dalmau, (iii) construcción del Centro Comercial Aeropuerto de El Salvador, (iv) construcción de gasolinera, y (v) construcción del cementerio Jardín Miraflores.

Al respecto podemos decir que aún falta ver los resultados de los proyectos de desarrollo que recién se están impulsando, que permitan a las industrias y comercios locales sostenerse y que logren competir en el mercado local, nacional y global.

\section{El Plan de la Comisión Nacional de Desarrollo (CND)}

La Comisión Nacional de Desarrollo presentó, en 1979, "El Plan de Nación", que propugna por una economía integradora que se base en la necesidad de desarrollar y articular la actividad económica de las diferentes zonas del país. El enfoque de este plan propone:

a) Basarse en premisas diferentes al concepto tradicional de "polos de desarrollo".

b) Plantea desarrollos regionales que no han sido planificados e impuestos desde las cúpulas gubernamentales o empresariales; al contrario, surgen del nivel local.

c) No se limita a la inversión de grandes capitales

d) No se limita al desarrollo de un solo sector

Las acciones están orientadas a la definición de "ejes integradores" y acciones iniciales más urgentes. La idea es dar solución a problemas en una dimensión más estructural, concreta y práctica. En las propuestas del Plan se toman en cuenta las condiciones sociopolíticas existentes, y considera que la mejor vía para desencadenar el desarrollo nacional, es ir de abajo hacia arriba, lo cual implica poner en marcha "regiones de desarrollo".

El plan propone cinco regiones, las cuales buscan generar una dinámica especial de desarrollo. Para la selección de estas regiones se tomaron en cuenta diversos aspectos: (i) los recursos naturales, analizando sus características y potencialidad económica y productiva; (ii) 
la disponibilidad de infraestructura física y de conectividad entre distintos municipios y departamentos; (iii) la densidad demográfica como factor importante para determinar a los beneficiarios directos e indirectos de acciones iniciales; y (iv) adicionalmente se ha considerado también la organización político/administrativa, la conectividad que la región posibilitaría con el mundo y el reordenamiento y equilibrio territorial que podría implicar en el futuro. También, han sido considerados distintos proyectos, estudios e iniciativas ya elaborados o en proceso de elaboración por otras instituciones.

Las regiones y los ejes de desarrollo propuestos son los siguientes:

o Región Oriental/Golfo de Fonseca: Puerta centroamericana al comercio internacional

o Región Occidental/Zona de los Volcanes: Desarrollo del ecoturismo nacional y centroamericano.

- Región Norte/Cuenca Alta del Río Lempa: Zona de producción de servicios ambientales

o Región Costera Central/Comalapa: Zona de innovación en exportación agrícola e industrial

o Región Metropolitana/Gran San Salvador: Transformación del sistema vial

El Plan de Nación propone para esta región dinamizar la oferta de exportación agrícola e industrial. La propuesta se orienta principalmente a conformar una zona de innovación modernizadora para la exportación de bienes industriales y agroindustriales. El centro de articulación lo ubican en el área de Comalapa - Olocuilta, que cuenta con una adecuada plataforma de techo industrial, servicios internacionales de transporte aéreo y la infraestructura inicial para el desarrollo del turismo marítimo.

La propuesta inicial es poner en acción a veinte y dos municipios pertenecientes a varios departamentos ${ }^{2}$, dentro de los cuales encontramos en el departamento de La Paz los municipios de Olocuilta y San Luis Talpa. (Comisión Nacional de Desarrollo,1999: 15-16, 2931).

Esta región cuenta con algunas de las mejores tierras cultivables, áreas de pastizales, montañas y marítima; sitios todos con potencial 
para la realización de actividades productivas complementarias del propósito industrial y de exportación para desarrollar el proyecto orientado hacia el mundo exterior.

El Aeropuerto Internacional de El Salvador (AIES), entró en operaciones en 1980, por la misma época se inició la construcción de la autopista para conectar el aeropuerto con la capital del país. La autopista de cuatro carriles con una capacidad para 3,000 vehículos/hora venía a sustituyó la carretera antigua. Antes de la construcción de la autopista, la región era básicamente de bosques de cafetal en la parte alta y cultivo de algodón en la parte baja.

La autopista se diseño como una vía rápida con accesos controlados y limitados a cinco lugares. Sin embargo, no se previó la demanda que generaría el crecimiento del Área Metropolitana de San Salvador (AMSS) y el tráfico que se generaría desde el oriente del país y el tráfico generado por las actividades del Aeropuerto.

Al inicio de la década de los ochenta, las condiciones de inversión se deterioran rápidamente. Esto afectó directamente la demanda de pasajeros en el Aeropuerto, al mismo tiempo que la inseguridad afectó la movilidad general. Ambos aspectos conjugados llevaron a que las expectativas en cuanto al volumen de tráfico en la autopista no alcanzara las proyecciones.

El tráfico internacional aeroportuario, que en 1978 había alcanzado un poco más de 357,000 pasajeros, para 1980 había decrecido en un 26\% a 264,015 pasajeros (VMVDU, 2000: 4). El volumen de pasajeros internacionales de entrada, que en 1978 había alcanzado un poco más de 180,000 para 1982 había decrecido en un $36 \%$ a 115,510 pasajeros.

Con la iluminación de la carretera en 1999 y la reconstrucción de la misma a través de la creación del Fondo Vial (FOVIAL) que inició sus actividades en el 2001, se espera una mayor reactivación de las actividades económicas en la región.

Esta región es la mejor conectada del país. La carretera litoral sirve para unir sitios claves, incluyendo la capital, playas, zonas productoras y áreas de vivienda. Cuenta, además, con buenas instalaciones eléctricas, telefónicas y la infraestructura adecuada para el funcionamiento de parques industriales. 
Se espera que las acciones propuestas por la CND tengan efectos desencadenantes en el desarrollo nacional porque se constituiría en una base sólida para el crecimiento de las exportaciones no tradicionales en el país, estableciendo una importante cadena productiva de alto valor agregado y generación de empleo, con efectos positivos para la integración productiva intersectorial (agropecuario, agroindustrial, industrial, servicios de transporte y comunicaciones).

Además el Plan de la CND busca contribuir a la desconcentración productiva y poblacional de la Región Metropolitana de San Salvador, tanto por la cercanía y fácil acceso a la capital como por la infraestructura instalada.

\section{Conclusiones}

La información anterior sostiene la tesis que tanto el municipio de Olocuilta y San Luis Talpa, por si mismos no hubieran sido capaces de despuntar, como lo están haciendo hasta ahora, sin un conjunto de políticas del Estado y la inversión tanto del sector privado como del público. Es de suma importancia indicar entonces, que para aquellas regiones donde las posibilidades de desarrollo real serán casi nulas, la reconversión pasa por la implementación de políticas y acciones estratégicas e integrales que vengan desde el Gobierno y la empresa privada.

El proceso de globalización en El Salvador, ha incidido y ha transformado las condiciones de vida (en el área rural y urbana). Según los indicadores de pobreza podría decirse que se han mejorado las condiciones de vida, pero según nuestro criterio sólo se han beneficiado ciertos sectores y no así los más pobres. Es indudable, que los países avanzados son los más beneficiados por este fenómeno y muy pocos son los beneficiados en los países en subdesarrollo. Es posible pensar que esto se deba principalmente, a las políticas y acciones aplicadas y a factores externos fuera de su control.

El Salvador como otros países pobres, no se puede permitir el lujo de quedar aislado de la economía mundial. Los avances logrados hasta la fecha en la economía del país no han sido suficientes y quizá no los más eficientes, pero a la vez no podemos dejar de darles su valor. Con esto queremos concluir que como consecuencia de las reformas y cambios estructurales en la economía del país, se están explorando nuevas

\section{7}

Globalización y desarrollo en la región de Olocuilto y San Luis Talpa 
formas de hacer gobierno, donde ya se reconoce a los gobiernos locales como los actores claves para el desarrollo económico de sus propias comunidades. Pero al mismo tiempo se hace necesario caminar hacia la creación y formación de alianzas con el gobierno, el sector de la empresa privada y otros sectores claves que permita a localidades como Olocuilta y San Luis Talpa impulsar un crecimiento económico sostenible.

La proximidad del parque industrial y habitacional en los municipios de Olocuilta y San Luis Talpa; la influencia de la autopista y el aeropuerto son elementos claves para estos municipios y la región. Y constituye uno de los grandes desafíos para el gobierno, en el sentido de generar ingresos crecientes de escala, a través de la aglomeración de empresas que permitan bajar los costos de producción y transacción.

Será de suma importancia en los próximos años desarrollar la información necesaria y disponible, así como el establecimiento de instituciones reguladoras y prácticas sociales como la concertación y apoyo que permitan mantener los costos de transacción a niveles bajos y potenciar las ventajas competitivas que ofrece la región.

\section{Referencias}

Borja, J. y Castells, M., (1997). Local y Global. La gestión de las ciudades en la era de la información, 32.

Carrera, A., (2001). Reforma del Estado y Descentralización, Diplomado en Gestión del Desarrollo Económico Local, FLACSO/IHS, Módulo 1.4, Sesión 4, Notas de clase.

Carrera, A., (2001). Evolución del proceso de reforma del Estado, Diplomado en Gestión del Desarrollo Económico Local, FLACSO/ IHS, Costa Rica, Módulo 1.4, Sesión 1 y 2, Notas de clase.

CEPAL/GTZ., (2000), Desarrollo Económico Local y Descentralización: aproximación a un marco conceptual, Santiago, Chile, 5.

Comisión Nacional de Desarrollo (1999). Acciones iniciales del Plan de Nación,_El Salvador, 15-16, 29-31.

DIGESTYC, (1992). Censo Nacional para el departamento de la Paz, El Salvador. 
Canal 12, (20 de diciembre, 2001). Programa de televisión, Frente a Frente.

De Campos, G. (2001). Planificando para regiones pobres en recursos en un mundo globalizante, 3.

García, N. (1999). La globalización imaginada, Argentina, 50.

La Prensa Gráfica. (2001). El Financiero. 4b.

La Reforma del Estado: Reto de la Democracia: www.clad.org.ve/ 0029803.html

Programa de las Naciones Unidas para el Desarrollo (PNUD), (2001). Informe sobre Desarrollo Humano, San Salvador, 92,95.

Perraton, J.; Goldblatt D.; Held D.; y McGrew A. (1997). The Globalisation of Economic Activity, 258.

PROESA (2001). Zonas Francas. Agencia de Promoción e Inversiones.

Syrkin, M., (1988). Patterns of Structural Change, Handbook of Development Economics, Volume I, Amsterdam, 205-228.

Sojo, C. (2001). La globalización y ajuste en América Latina y en Centro América, Diplomado en Gestión del Desarrollo Económico Local, FLACSO/IHS, Costa Rica, Módulo I, Notas de clase.

UCA, Departamento de Economía (2001). La verdad secuestrada. Estudios Centroamericanos (ECA), El Salvador, 794.

VMVDU, Dirección de Desarrollo Urbano y Regional, (2000). Estudio de desarrollo físico a nivel de diagnóstico para la zona de influencia de la autopista San Salvador-Aeropuerto Internacional El Salvador, San Salvador, 4, 48, 56.

\section{Notas}

1. "El ILE parte de una concepción limitada de las libertades que lo vuclve incongruente con el paradigma del desarrollo humano. Por ejemplo, un bajo nivel de gasto público, de acuerdo a cste índice, mejora las libertades económicas, debido a que refleja poca intervención del Estado, independientemente de que, como consecuencia de ello, se limite el papel redistributivo de la política fiscal. El ILE tampoco considera la igualdad de oportunidades ni la libertad de carencias físicas, como el hambre, las cuales son esenciales dentro del paradigma del desarrollo humano". 
2. Departamento de La Paz: San Luis, San Juan Talpa, San Luis de la Herradura, El Rosario, Olocuilta, Cuyultitán, Tapalhuaca, San Pedro Masahuat, Santiago Nonualco, San Juan Nonualco y Zacatecoluca; Departamento de San Vicente: Tecoluca, Guadalupe y San Vicente (cabecera); Departamento de La Libertad: Puerto de La Libertad, San José Villanueva y Zaragoza; Departamento de Cuscatlán: Cojutepeque, San Ramón y Santa Cruz Analquito; Departamento de Cabañas: Tejutepeque e Ilobasco. 